\title{
Heat capacity and spin susceptibility of two-dimensional $t-J$ model
}

\author{
T.N. Antsygina, M.I. Poltavskaya, I.I. Poltavsky, and K.A. Chishko \\ B. Verkin Institute for Low Temperature Physics and Engineering of the National Academy of Sciences of Ukraine \\ 47 Lenin Ave., Kharkov 61103, Ukraine \\ E-mail: chishko@ilt.kharkov.ua \\ Received October 20, 2006
}

\begin{abstract}
Thermodynamic properties of the $t-J$ model on square and triangular lattices near half-filling are investigated theoretically within an analytical approach based on the Kondo and Yamaji's Green function decoupling scheme. The temperature dependences of the heat capacity and spin susceptibility are calculated in the wide temperature range for the case when the exchange constant $J$ is greater than the hopping amplitude $t$. It was found, that with the increase of doping from the half-filling, the maximum of the spin susceptibility increases and its position shifts to lower temperatures for both types of lattices. Such behavior is in agreement with the qualitative predictions [E. Dagotto, Rev. Mod. Phys. 66, 763 (1994)]. Heat capacity demonstrates a double peak shape. The high temperature peak associated with the «spin wave-like» excitations shifts to lower temperatures with doping. The low temperature peak appears due to the holes and its height and position depend on both the doping and the ratio $t / J$.
\end{abstract}

PACS: 71.10.Fd Lattice fermion models (Hubbard model, etc.);

75.40.Cx Static properties (order parameter, static susceptibility, heat capacities, critical exponents, etc.).

Keywords: two-dimensional $t-J$ model, heat capacity, susceptibility.

\section{Introduction}

The $t-J$ model is one of the central models for strongly correlated systems. It has been studied intensively in various theoretical aspects and also in view of its applications to the treatment of experimentally observed phenomena [1-3]. Of special interest is the two-dimensional $t-J$ model on square and triangular lattices.

As compared to the triangular lattice, the square-lattice $t-J$ model has been investigated in much more detail for at least two reasons. First, the theoretical results obtained with this model are of principal importance to explain the real features of high-temperature superconductivity. These results appeared to be useful for a better understanding of the normal state properties of high- $T_{c}$ superconductors [1] as well as of the mechanism of hole-type high- $T_{c}$ superconductivity [4]. Second, the theoretical treatment of the frustrated $t-J$ model on a triangular lattice in some respects is more complicated.

Most efforts were directed to the study of the ground state of the square-lattice $t-J$ model, and also its hightemperature properties are carefully investigated at least for $t / J>1[5,6]$. In the intermediate temperature range the model was studied numerically $[1,3]$, and analytical results were obtained for $t / J>2.5$ [2]. In Ref. 7 the Green function formalism with the extended Kondo-Yamaji decoupling procedure [8] has been employed to describe the dependences of the magnetization and magnetic susceptibility upon the hole concentration at $T=0$ and $t<<J$. This theory has been constructed in terms of quantities describing a short-range order, i.e., in terms of correlation functions for which a system of selfconsistent equations has been derived.

Theoretically, the $t-J$ model on the triangular lattice is less investigated. By now, as far as we know, it was treated only within high-temperature series expansions $[9,10]$. On the other hand, it has been suggested recently $[11,12]$ that the triangular lattice $t-J$ model can be appropriate to interpret the behavior of the heat capacity in solid ${ }^{3} \mathrm{He}$ layers on graphite substrates. Thus, having in view the practical application of the theoretical results it is important to develop analytical approaches to describe the thermodynamics of the model at arbitrary temperatures.

In the present work, on the basis of the analytical method [7] we study thermodynamic properties (temperature 
dependences of the magnetic susceptibility and heat capacity) of the $t-J$ model on the triangular and square lattices near half-filling for $t / J<<1$ in the wide temperature range. We take into account some fluctuation terms omitted in Ref. 7. In our calculation scheme the systems of self-consistent equations have an identical form for both types of lattices, differing only in the form of the structural factor entering these equations.

\section{Model}

We consider an assembly of $N_{p}$ spins $S=1 / 2$ on the two-dimensional lattice with $N$ sites. The Hamiltonian has the form

$$
H=H_{t}+H_{J}
$$

Here

$$
H_{t}=-t \sum_{\mathbf{f}, \delta, \sigma} \widetilde{c}_{\mathbf{f}, \sigma}^{+} \widetilde{c}_{\mathbf{f}}+\delta, \sigma,
$$

where $t$ is the matrix element of the hopping between nearest neighbour sites, $\delta$ is the unit vector connecting the nearest neighbours. The annihilation operator $\widetilde{c}_{\mathbf{f}, \sigma}$ is defined through the conventional Fermi-operators $c_{\mathbf{f}, \sigma}, c_{\mathbf{f}, \sigma}^{+}$ as

$$
\tilde{c}_{\mathbf{f}, \sigma}=c_{\mathbf{f}, \sigma}\left(1-n_{\mathbf{f}, \bar{\sigma}}\right), n_{\mathbf{f}, \sigma}=c_{\mathbf{f}, \sigma}^{+} c_{\mathbf{f}, \sigma}, \bar{\sigma}=-\sigma,
$$

so that the double occupation of the lattice sites is forbidden. The second term in (1) is

$$
H_{J}=\frac{J}{2} \sum_{\mathbf{f}, \delta}\left(\mathbf{S}_{\mathbf{f}} \mathbf{S}_{\mathbf{f}+\delta}-\frac{1}{4} n_{\mathbf{f}} n_{\mathbf{f}+\delta}\right),
$$
where $J$ is the exchange constant, $n_{\mathbf{f}}=\sum_{\sigma} n_{\mathbf{f}, \sigma}, \mathbf{S}_{\mathbf{f}}$ is the
spin operator,

$$
\begin{aligned}
& S_{\mathbf{f}}^{x}=\frac{1}{2} \sum_{\sigma} c_{\mathbf{f}, \sigma}^{+} c_{\mathbf{f}, \bar{\sigma}}, \\
& S_{\mathbf{f}}^{y}=-\frac{i}{2} \sum_{\sigma} \sigma c_{\mathbf{f}, \sigma}^{+} c_{\mathbf{f}, \bar{\sigma}}, . \\
& S_{\mathbf{f}}^{z}=\frac{1}{2} \sum_{\sigma} \sigma n_{\mathbf{f}, \sigma} .
\end{aligned}
$$

We consider the practically important case: $n=\left\langle n_{\mathbf{f}}\right\rangle=$ $=N_{p} / N$ is close to unity, and the number of holes $n_{h}=$ $=1-n$ is small. We also restrict our consideration to $t<<J$.

\section{Method}

The calculation method we use is based on the formalism of two-time Green functions. Since in the systems under consideration the long-range order does not exist at any finite temperature, the problem should be treated in terms of local correlations describing a short range order.
We need two types of Green functions: two-spin $\left\langle\left\langle S_{\mathbf{f}}^{z} \mid S_{\mathbf{f}^{\prime}}^{z}\right\rangle\right\rangle$ and one-particle $\left\langle\left\langle\widetilde{c}_{\mathbf{f}, \sigma} \mid \widetilde{c}_{\mathbf{f}^{\prime}, \sigma}^{+}\right\rangle\right\rangle$.

Let us start with the two-spin Green function whose Fourier time transform satisfies the equation of motion

$$
\begin{gathered}
i \omega\left\langle\left\langle S_{\mathbf{f}}^{z} \mid S_{\mathbf{f}^{\prime}}^{z}\right\rangle\right\rangle_{\omega}= \\
=J \sum_{\delta}\left(\left\langle\left\langle S_{\mathbf{f}}^{x} S_{\mathbf{f}+\delta}^{y} S_{\mathbf{f}^{\prime}}^{z}\right\rangle\right\rangle_{\omega}-\left\langle\left\langle S_{\mathbf{f}}^{y} S_{\mathbf{f}+\delta}^{x} \mid S_{\mathbf{f}^{\prime}}^{z}\right\rangle\right\rangle_{\omega}\right)- \\
-\frac{i t}{2} \sum_{\delta, \sigma} \sigma\left(\left\langle\left\langle\widetilde{c}_{\mathbf{f}, \sigma}^{+} \widetilde{c}_{\mathbf{f}+\delta, \sigma} \mid S_{\mathbf{f}^{\prime}}^{z}\right\rangle\right\rangle_{\omega}-\left\langle\left\langle\widetilde{c}_{\mathbf{f}+\delta, \sigma}^{+} \widetilde{c}_{\mathbf{f}, \sigma} \mid S_{\mathbf{f}^{\prime}}^{z}\right\rangle\right\rangle_{\omega}\right) .
\end{gathered}
$$

At this stage the decoupling of the higher functions does not lead to any satisfactory results because the average value of the spin operator equals to zero. That is why it is necessary to put down the equations of motion for the Green functions in the right hand side of (6). Then we decouple the higher Green functions appearing in the right hand sides of the obtained equations. We follow the decoupling procedure proposed in [7] which is the extension of the Kondo and Yamaji approach [8] originally developed for low-dimensional Heisenberg systems with spin $1 / 2$. According to this procedure the operators on the same lattice site are not decoupled in order to retain the local correlations. This approach is believed to give a proper description for the systems under study [7].

The equation of motion for the first term in the right hand side of (6) has the form

$$
\begin{gathered}
i \omega\left(\left\langle\left\langle S_{\mathbf{f}}^{x} S_{\mathbf{f}+\delta}^{y} \mid S_{\mathbf{f}^{\prime}}^{z}\right\rangle\right\rangle_{\omega}-\left\langle\left\langle S_{\mathbf{f}}^{y} S_{\mathbf{f}+\delta}^{x} \mid S_{\mathbf{f}^{\prime}}^{z}\right\rangle\right\rangle_{\omega}\right)= \\
=-\frac{c_{1}}{4} \pi\left(\Delta_{\mathbf{f}+\delta, \mathbf{f}^{\prime}}-\Delta_{\mathbf{f}, \mathbf{f}^{\prime}}\right)+ \\
+J \sum_{\delta^{\prime}, v}\left(\left\langle\left\langle S_{\mathbf{f}}^{v} S_{\mathbf{f}+\delta^{\prime}}^{z} S_{\mathbf{f}+\delta}^{\nu} \mid S_{\mathbf{f}^{\prime}}^{z}\right\rangle\right\rangle_{\omega}-\left\langle\left\langle S_{\mathbf{f}}^{z} S_{\mathbf{f}+\delta^{\prime}}^{\nu} S_{\mathbf{f}+\delta}^{\nu} \mid S_{\mathbf{f}^{\prime}}^{z}\right\rangle\right\rangle_{\omega}+\right. \\
\left.+\left\langle\left\langle S_{\mathbf{f}}^{\nu} S_{\mathbf{f}+\delta}^{z} S_{\mathbf{f}+\delta-\delta^{\prime}}^{\nu} \mid S_{\mathbf{f}^{\prime}}^{z}\right\rangle\right\rangle_{\omega}-\left\langle\left\langle S_{\mathbf{f}}^{\nu} S_{\mathbf{f}+\delta}^{\nu} S_{\mathbf{f}+\delta-\delta^{\prime}}^{z} \mid S_{\mathbf{f}^{\prime}}^{z}\right\rangle\right\rangle_{\omega}\right)+ \\
+i\left\langle\left\langle\left[\left(S_{\mathbf{f}}^{x} S_{\mathbf{f}+\delta}^{y}-S_{\mathbf{f}}^{y} S_{\mathbf{f}+\delta}^{x}\right), H_{t}\right] S_{\mathbf{f}^{\prime}}^{z}\right\rangle\right\rangle_{\omega}, v=x, y, \quad \text { (7) }
\end{gathered}
$$

where the correlation function

$$
c_{1}=4\left\langle S_{\mathbf{f}}^{\nu} S_{\mathbf{f}+\delta}^{\nu}\right\rangle, v=x, y, z
$$

describes spin correlations between the particles at the nearest neighbour sites. We illustrate the decoupling procedure using the first term in the sum over $\boldsymbol{\delta}^{\prime}$ with $v=y$. It can be rewritten in the form

$$
\begin{aligned}
& \left\langle\left\langle S_{\mathbf{f}}^{y} S_{\mathbf{f}+\delta^{\prime}}^{z} S_{\mathbf{f}+\delta}^{y} \mid S_{\mathbf{f}^{\prime}}^{z}\right\rangle\right\rangle_{\omega}=-\frac{i}{2} \Delta_{\delta, \delta^{\prime}}\left\langle\left\langle S_{\mathbf{f}}^{y} S_{\mathbf{f}+\delta}^{x} \mid S_{\mathbf{f}^{\prime}}^{z}\right\rangle\right\rangle_{\omega}+ \\
& +\left(1-\Delta_{\delta, \delta^{\prime}}\right)\left\langle\left\langle S_{\mathbf{f}}^{y} S_{\mathbf{f}+\delta}^{z} S_{\mathbf{f}+\delta^{\prime}}^{y} \mid S_{\mathbf{f}^{\prime}}^{z}\right\rangle\right\rangle_{\omega},
\end{aligned}
$$


where $\Delta_{i, j}$ is the Kronecker symbol. According to [7,8] we have for the Green functions containing only spin operators on different sites (i.e. $\boldsymbol{\delta} \neq \boldsymbol{\delta}^{\prime}$ )

$$
\begin{aligned}
& \left\langle\left\langle S_{\mathbf{f}}^{y} S_{\mathbf{f}+\delta}^{z} S_{\mathbf{f}+\delta^{\prime}}^{y} \mid S_{\mathbf{f}^{\prime}}^{z}\right\rangle\right\rangle_{\omega} \rightarrow \alpha\left\langle S_{\mathbf{f}}^{y} S_{\mathbf{f}+\delta^{\prime}}^{y}\right\rangle\left\langle\left\langle S_{\mathbf{f}+\delta}^{z} \mid S_{\mathbf{f}^{\prime}}^{z}\right\rangle\right\rangle_{\omega}= \\
& =\frac{\tilde{c}_{1}}{4}\left\langle\left\langle S_{\mathbf{f}+\delta}^{z} \mid S_{\mathbf{f}^{\prime}}^{z}\right\rangle\right\rangle_{\omega} .
\end{aligned}
$$

Here $\widetilde{c}_{1}=\alpha c_{1}$. A vertex parameter $\alpha$ is a function of temperature and is chosen to satisfy the sum rule

$$
\left\langle\left(S_{\mathbf{f}}^{\vee}\right)^{2}\right\rangle=\frac{\left\langle n_{\mathbf{f}}\right\rangle}{4}=\frac{n}{4} .
$$

In the same manner we treat other terms in the sum over $\delta^{\prime}$ in Eq. (7). The last term in (7) vanishes after decoupling. As a result, we have

$$
\begin{gathered}
i \omega \sum_{\delta}\left(\left\langle\left\langle S_{\mathbf{f}}^{x} S_{\mathbf{f}+\delta}^{y} \mid S_{\mathbf{f}^{\prime}}^{z}\right\rangle\right\rangle_{\omega}-\left\langle\left\langle S_{\mathbf{f}}^{y} S_{\mathbf{f}+\delta}^{x} \mid S_{\mathbf{f}^{\prime}}^{z}\right\rangle\right\rangle_{\omega}\right)=-\frac{c_{1}}{4 \pi} \sum_{\delta}\left(\Delta_{\mathbf{f}+\delta, \mathbf{f}^{\prime}}-\Delta_{\mathbf{f}, \mathbf{f}^{\prime}}\right)+ \\
+\frac{J}{2}\left[\left(n+\widetilde{c}_{2}\right) \sum_{\delta}\left(\left\langle\left\langle S_{\mathbf{f}+\delta}^{z} \mid S_{\mathbf{f}^{\prime}}^{z}\right\rangle\right\rangle_{\omega}-\left\langle\left\langle S_{\mathbf{f}}^{z} \mid S_{\mathbf{f}^{\prime}}^{z}\right\rangle\right\rangle_{\omega}\right)-\widetilde{c}_{1} \sum_{\delta, \delta^{\prime}}^{\prime}\left(\left\langle\left\langle S_{\mathbf{f}+\delta-\delta^{\prime}}^{z} \mid S_{\mathbf{f}^{\prime}}^{z}\right\rangle\right\rangle_{\omega}-\left\langle\left\langle S_{\mathbf{f}+\delta}^{z} \mid S_{\mathbf{f}^{\prime}}^{z}\right\rangle\right\rangle_{\omega}\right)\right] .
\end{gathered}
$$

Here a prime over the sum stands for $\delta \neq \delta^{\prime}$ and a linear combination

$$
c_{2}=4 \sum_{\delta^{\prime}}^{\prime}\left\langle S_{\mathbf{f}+\delta}^{v} S_{\mathbf{f}+\delta^{\prime}}^{\nu}\right\rangle, \quad \tilde{c}_{2}=\alpha c_{2}, \quad v=x, y, z
$$

describes correlations between spins which are two steps apart along the translation vector.

Let us now proceed to the second term in the right hand side of (6). Being proportional to $t$ this term vanishes in the half-filling limit $n \rightarrow 1$ and its contribution to the two-spin Green function is small. That is why in the equation of motion for this term we perform decoupling without introducing any vertex corrections. Thus, we obtain

$$
\begin{gathered}
\omega \sum_{\delta, \sigma} \sigma\left(\left\langle\left\langle\widetilde{c}_{\mathbf{f}, \sigma}^{+} \widetilde{c}_{\mathbf{f}+\delta, \sigma} \mid S_{\mathbf{f}^{\prime}}^{z}\right\rangle\right\rangle_{\omega}-\left\langle\left\langle\widetilde{c}_{\mathbf{f}+\delta, \sigma}^{+} \widetilde{c}_{\mathbf{f}, \sigma} \mid S_{\mathbf{f}^{\prime}}^{z}\right\rangle\right\rangle_{\omega}\right)=\frac{n_{1}}{\pi} \sum_{\delta}\left(\Delta_{\mathbf{f}+\delta, \mathbf{f}^{\prime}}-\Delta_{\mathbf{f}, \mathbf{f}^{\prime}}\right)+ \\
+2 J n_{1} \sum_{\delta, \delta^{\prime}}^{\prime}\left(\left\langle\left\langle S_{\mathbf{f}+\delta-\delta^{\prime}}^{z} \mid S_{\mathbf{f}^{\prime}}^{z}\right\rangle\right\rangle_{\omega}-\left\langle\left\langle S_{\mathbf{f}+\delta}^{z} \mid S_{\mathbf{f}^{\prime}}^{z}\right\rangle\right\rangle_{\omega}\right)+4 t\left(n_{h}-n_{2}\right) \sum_{\delta}\left(\left\langle\left\langle S_{\mathbf{f}+\delta}^{z} \mid S_{\mathbf{f}^{\prime}}^{z}\right\rangle\right\rangle_{\omega}-\left\langle\left\langle S_{\mathbf{f}}^{z} \mid S_{\mathbf{f}^{\prime}}^{z}\right\rangle\right\rangle_{\omega}\right)+ \\
+t \bar{n} \sum_{\delta, \delta^{\prime}, \sigma}^{\prime}\left(2\left\langle\left\langle\widetilde{c}_{\mathbf{f}+\delta, \sigma}^{+} \widetilde{c}_{\mathbf{f}+\delta^{\prime}, \sigma} \mid S_{\mathbf{f}^{\prime}}^{z}\right\rangle\right\rangle_{\omega}-\left\langle\left\langle\widetilde{c}_{\mathbf{f}, \sigma}^{+} \widetilde{c}_{\mathbf{f}+\delta-\delta^{\prime}, \sigma} \mid S_{\mathbf{f}^{\prime}}^{z}\right\rangle\right\rangle_{\omega}-\left\langle\left\langle\widetilde{c}_{\mathbf{f}+\delta-\delta^{\prime}, \sigma}^{+} \widetilde{c}_{\mathbf{f}, \sigma} \mid S_{\mathbf{f}^{\prime}}^{z}\right\rangle\right\rangle_{\omega}\right) .
\end{gathered}
$$

Here the following notifications are introduced

$$
\begin{aligned}
& n_{1}=\left\langle\tilde{c}_{\mathbf{f}, \sigma}^{+} \tilde{c}_{\mathbf{f}+\delta, \sigma}\right\rangle, \\
& \bar{n}=1-\frac{n}{2}, n_{2}=\sum_{\delta^{\prime}}^{\prime}\left\langle\widetilde{c}_{\mathbf{f}+\delta, \sigma}^{+} \widetilde{c}_{\mathbf{f}+\delta^{\prime}, \sigma}\right\rangle .
\end{aligned}
$$

It can be shown that the last term in the right hand side of Eq. (10) is less than other terms at least by a factor of $t / J$ and can be neglected.

Substituting the expressions (9) and (10) into Eq. (6) we obtain the closed equation for the two-spin Green function, which includes the correlation functions $c_{1}, c_{2}$, $n_{1}, n_{2}$ and the vertex parameter $\alpha$ to be found. For the Fourier transform in coordinates of the two-spin Green function we have

$$
\left\langle\left\langle S_{\mathbf{k}}^{z} \mid S_{-\mathbf{k}}^{z}\right\rangle\right\rangle_{\omega}=\frac{2 t n_{1}-J c_{1}}{4 \pi} \frac{\gamma_{0}-\gamma_{\mathbf{k}}}{\omega^{2}-\omega_{\mathbf{k}}^{2}},
$$

where

$$
\begin{gathered}
\omega_{\mathbf{k}}^{2}=\frac{J^{2}}{2}\left(\gamma_{0}-\gamma_{\mathbf{k}}\right)\left[\Delta-\left(\gamma_{0}+\gamma_{\mathbf{k}}\right)\left(\widetilde{c}_{1}-\frac{2 t}{J} n_{1}\right)\right], \\
\Delta=n+\left(\gamma_{0}-1\right)\left(\widetilde{c}_{1}-\frac{2 t}{J} n_{1}\right)+\widetilde{c}_{2}+\left(\frac{2 t}{J}\right)^{2}\left(n_{h}-n_{2}\right),
\end{gathered}
$$

the structural factor $\gamma_{\mathbf{k}}$ is determined by

$$
\gamma_{\mathbf{k}}=\sum_{\delta} \exp (i \mathbf{k} \delta),
$$

and $\gamma_{0}$ is a coordination number.

The equation of motion for the Green function $\left\langle\left\langle\widetilde{c}_{\mathbf{f}, \sigma} \mid \widetilde{c}_{\mathbf{f}^{\prime}, \sigma}^{+}\right\rangle\right\rangle$has the form 


$$
\begin{aligned}
& \omega\left\langle\left\langle\widetilde{c}_{\mathbf{f}, \sigma} \mid \widetilde{c}_{\mathbf{f}^{\prime}, \sigma}^{+}\right\rangle\right\rangle_{\omega}=\frac{\bar{n}}{2 \pi} \Delta_{\mathbf{f}, \mathbf{f}^{\prime}}-t \sum_{\delta}\left[\left\langle\left\langle\left(1-n_{\mathbf{f}, \bar{\sigma}}\right) \widetilde{c}_{\mathbf{f}+\delta, \sigma} \mid \widetilde{c}_{\mathbf{f}^{\prime}, \sigma}^{+}\right\rangle\right\rangle_{\omega}+\left\langle\left\langle S_{\mathbf{f}}^{\bar{\sigma}} \widetilde{c}_{\mathbf{f}+\delta, \bar{\sigma}} \mid \widetilde{c}_{\mathbf{f}^{\prime}, \sigma}^{+}\right\rangle\right\rangle_{\omega}\right]+ \\
& \quad+\frac{J}{2} \sum_{\delta}\left[\left\langle\left\langle S_{\mathbf{f}+\delta}^{\bar{\sigma}} \widetilde{c}_{\mathbf{f}, \bar{\sigma}} \mid \widetilde{c}_{\mathbf{f}^{\prime}, \sigma}^{+}\right\rangle\right\rangle_{\omega}+\sigma\left\langle\left\langle S_{\mathbf{f}+\delta}^{z} \widetilde{c}_{\mathbf{f}, \sigma} \mid \widetilde{c}_{\mathbf{f}^{\prime}, \sigma}^{+}\right\rangle\right\rangle_{\omega}-\frac{1}{2}\left\langle\left\langle n_{\mathbf{f}+\delta} \widetilde{c}_{\mathbf{f}, \sigma} \mid \widetilde{c}_{\mathbf{f}^{\prime}, \sigma}^{+}\right\rangle\right\rangle_{\omega}\right],
\end{aligned}
$$

where $S_{\mathbf{f}}^{\sigma}=S_{\mathbf{f}}^{x}+i \sigma S_{\mathbf{f}}^{y}$. We treat (14) in a similar way as the second term in (6). In the main approximation we get for the Fourier transform $\left\langle\left\langle\widetilde{c}_{\mathbf{k}, \sigma} \mid \widetilde{c}_{-\mathbf{k}, \sigma}^{+}\right\rangle\right\rangle_{\omega}$

$$
\left\langle\left\langle\widetilde{c}_{\mathbf{k}, \sigma} \mid \widetilde{c}_{-\mathbf{k}, \sigma}^{+}\right\rangle\right\rangle_{\omega}=\frac{\bar{n}}{2 \pi\left(\omega-\Omega_{\mathbf{k}}\right)}, \Omega_{\mathbf{k}}=-t \bar{n} \gamma_{\mathbf{k}}-\frac{J}{4} \gamma_{0} n .
$$

This approximation is rather crude and the functions $n_{1}$, $n_{2}$ calculated within it should be considered only as reasonable estimates. A more elaborate result for the Green function (15) can be obtained by accounting for fluctuations of order $J$ in the right hand side of (14).

Using the dispersion relations for the Green functions, we have:

$$
\begin{gathered}
4\left\langle S_{\mathbf{k}}^{z} S_{-\mathbf{k}}^{z}\right\rangle=\left(2 t n_{1}-J c_{1}\right) \frac{\gamma_{0}-\gamma_{\mathbf{k}}}{\omega_{\mathbf{k}}} \operatorname{coth} \frac{\omega_{\mathbf{k}}}{2 T}, \\
\left\langle\widetilde{c}_{\mathbf{k}, \sigma}^{+} \widetilde{c}_{-\mathbf{k}, \sigma}\right\rangle=\bar{n}\left[\exp \left(\frac{\Omega_{\mathbf{k}}-\mu}{T}\right)+1\right]^{-1},
\end{gathered}
$$

where $\mu$ is the chemical potential. Finally, we find the following complete set of equations:

$$
\begin{gathered}
\frac{4}{N} \sum_{\mathbf{k}}\left\langle S_{\mathbf{k}}^{z} S_{-\mathbf{k}}^{z}\right\rangle=n, \frac{2}{N} \sum_{\mathbf{k}}\left\langle\widetilde{c}_{\mathbf{k}, \sigma}^{+} \widetilde{c}_{-\mathbf{k}, \sigma}\right\rangle=n, \\
\frac{4}{N} \sum_{\mathbf{k}} \frac{\gamma_{\mathbf{k}}}{\gamma_{0}}\left\langle S_{\mathbf{k}}^{z} S_{-\mathbf{k}}^{z}\right\rangle=c_{1}, \frac{4}{N} \sum_{\mathbf{k}}\left(\frac{\gamma_{\mathbf{k}}^{2}}{\gamma_{0}}-1\right)\left\langle S_{\mathbf{k}}^{z} S_{-\mathbf{k}}^{z}\right\rangle=c_{2}, \\
\frac{1}{N} \sum_{\mathbf{k}} \frac{\gamma_{\mathbf{k}}}{\gamma_{0}}\left\langle\widetilde{c}_{\mathbf{k}, \sigma}^{+} \widetilde{c}_{-\mathbf{k}, \sigma}\right\rangle=n_{1}, \frac{1}{N} \sum_{\mathbf{k}}\left(\frac{\gamma_{\mathbf{k}}^{2}}{\gamma_{0}}-1\right)\left\langle\widetilde{c}_{\mathbf{k}, \sigma}^{+} \widetilde{c}_{-\mathbf{k}, \sigma}\right\rangle=n_{2} .
\end{gathered}
$$

The first equation is due to the sum rule (8), the second one determines the chemical potential. Other equations in (18) arise from the definitions of $c_{1}, c_{2}$ and $n_{1}, n_{2}$. In the general case the system (18) can be solved only numerically. The heat capacity per one site and static spin susceptibility are calculated using the following expressions:

$$
\begin{aligned}
& \frac{C_{v}}{N}=\gamma_{0}\left(\frac{3 J}{8} \frac{d c_{1}}{d T}-2 t \frac{d n_{1}}{d T}\right), \\
& \chi=\frac{2 t n_{1}-J c_{1}}{J\left[J \Delta+2 \gamma_{0}\left(2 t n_{1}-\widetilde{c}_{1}\right)\right]} .
\end{aligned}
$$

\section{Results and discussion}

In our theory the Heisenberg limit is correctly recovered because $n_{1}, n_{2}$ tend to zero with $n \rightarrow 1$. Near the half-filling, the functions $n_{1}, n_{2}$ are small quantities of order $1-n$.

The decoupling scheme used leads to the Green functions corresponding to two types of elementary excitations. The «spin wave»-like excitations are described in terms of spin operators. The excitations of the second type associated with the correlated motion of the holes are described by means of the one-particle Green function. The «spin wave»-like excitations exist due to the condition $n_{h}<<1$. Another restriction, $t<<J$, means that the spin excitations are faster than the excitations of the second type. That is why the two types of excitations are effectively separated. Owing to this fact it is possible to obtain a closed equation for the two-spin Green function (see (6), (10)). As can be seen from (12) and (13), the frequencies of the spin waves differ slightly from those for the pure Heisenberg system, because of the inequalities $n_{h}<<1, t<<J$.

Temperature dependences of the spin susceptibility and heat capacity per lattice site are shown in Fig. 1 (for the square lattice) and Fig. 2 (for the triangular lattice). The Heisenberg limit is shown as solid curves. Curves 1 , 2 correspond to $n=0.95, t / J=0.1$ and curves 3,4 - to $n=0.95, t / J=0.25$. Curves 1,3 were calculated using Eq. (15), and curves 2, 4 were obtained by more accurate calculations with account for the fluctuation terms of or$\operatorname{der} \sim J$ in Eq. (14).

Let us discuss $\chi(T)$. It was mentioned [1] that for the $t-J$ model on the square lattice the spin susceptibility maximum increases in amplitude and shifts to lower temperatures with increasing doping. It was suggested [1] that this statement holds true for arbitrary values of $t / J$, and, furthermore, the zero temperature susceptibility increases with doping. These facts were qualitatively explained in the following way. Due to the presence of holes in the doped Heisenberg antiferromagnet the exchange frequency $J$ is effectively reduced. As a consequence, the temperature where $\chi(T)$ has a maximum decreases, and the zero temperature susceptibility increases because $\chi \sim 1 / J$. As can be seen from Fig. 1, $a$, all these features in $\chi(T)$ behavior are reproduced by our theory. The same qualitative picture holds for the triangular lattice (see Fig. 2,a), but the height of the peak depends on the ratio $t / J$ stronger than for the square lattice. As $T / J>>1$ the 

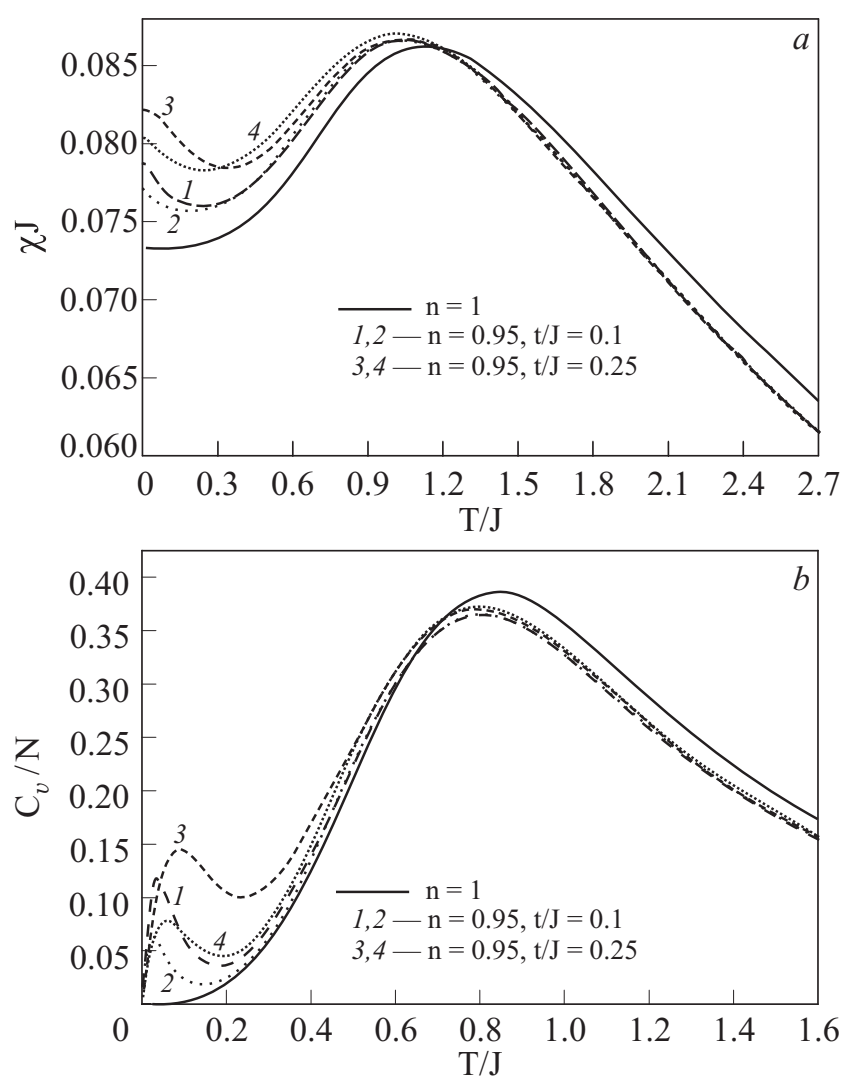

Fig. 1. Spin susceptibility $(a)$ and heat capacity (b) for the square lattice. Curves 1, 3 are calculated using Eq. (15), curves 2, 4 are calculated with account for fluctuation terms in Eq. (14).

asymptotics for $\chi$ has the form $\chi=n /(4 T)$, so that the susceptibility is independent of $t / J$.

Now we proceed to the temperature dependence of the heat capacity. The main result obtained here is the double-peak shape of $C_{v}(T)$. The high temperature peak is mainly due to the «spin wave»-like excitations. Under doping this peak shifts to lower temperatures. The low temperature peak in $C_{v}(T)$ curves is due to the hole motion. Its height and position depend appreciably on both the doping and the $t / J$ ratio. Note that for the triangular lattice the hole motion gives a noticeable contribution to the amplitude of the high temperature peak. When $T \rightarrow \infty$ the heat capacity decreases as

$$
\frac{C_{v}}{N}=\gamma_{0} \frac{n}{4}\left[\frac{3}{8} n+\left(\frac{2 t}{J}\right)^{2} n_{h}\right]\left(\frac{J}{T}\right)^{2} .
$$

As $T \rightarrow 0$, the heat capacity is linear in temperature. Our results for $C_{v}(T)$ agree qualitatively with the numerical calculations [3] for the cluster on the square lattice with 10 sites. It can be seen from Figs. 1 and 2, that fluctuations affect low temperature run of $C_{v}(T)$ and $\chi(T)$ (curves 2,4). These changes are more pronounced for
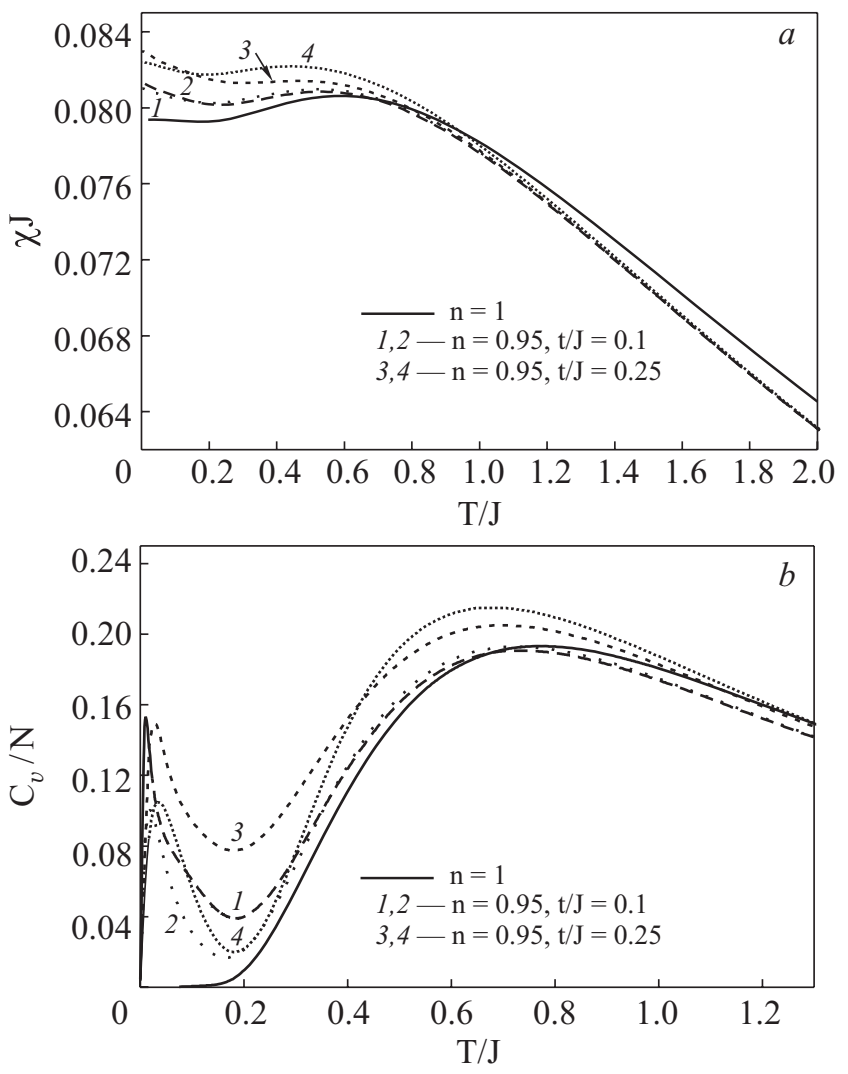

Fig. 2. Spin susceptibility (a) and heat capacity (b) for the triangular lattice. Curves 1,3 are calculated using Eq. (15), curves 2, 4 are calculated with account for fluctuation terms in Eq. (14).

$C_{v}(T)$ because the expression (19) for $C_{v}$ contains the derivatives of $n_{1}$ and $c_{1}$ with respect to $T$.

Using $C_{v}(T)$ we obtain the entropy

$$
S(T)=\int_{0}^{T} \frac{C_{v}(T)}{T} d T .
$$

The limiting value of the entropy per site at $T \rightarrow \infty$ is

$$
\frac{S(\infty)}{N}=-\left[n \ln \left(\frac{n}{2}\right)+n_{h} \ln n_{h}\right] .
$$

The values of $S(\infty) / N$ calculated from the dependences in Figs. $1, b$ and $2, b$ are close to the corresponding exact values. For example, for the triangular lattice with $t / J=0.25$ the entropy at $T \rightarrow \infty$ is 0.831 for curve 3 and 0.667 for curve 4 while the exact value is $S(\infty) / N=0.857$. In all cases the entropies calculated for curves 2, 4 are less than those calculated for curves 1,3 . This fact seems to be natural, because within the Kondo-Yamaji approach the entropy of a pure antiferromagnet is appreciably underestimated. 
1. E. Dagotto, Rev. Mod. Phys. 66, 763 (1994).

2. S. Winterfeldt and D. Ihle, Phys. Rev. B58, 9402 (1998).

3. H. Röder, H. Fehske, V. Waas, and H. Büttner, Phys. Rev. B45, 13092 (1992).

4. P.A. Lee, in: Mechanism of High Temperature Conductivity, v. II, H. Kamimura and A. Oshiyama (eds.), Springer-Verlag, Berlin (1989).

5. W.O. Putikka, R.L. Glenister, R.R.P. Singh, and H. Tsunetsugu, cond-mat/9309031.

6. W.O. Putikka, M.U. Luchini, and R.R.P. Singh, condmat/9912269.

7. H. Shimahara and S. Takada, J. Phys. Soc. Jpn. 61, 989 (1992).

8. J. Kondo and K. Yamaji, Progr. Theor. Phys. 47, 807 (1972).
9. T. Koretsune and M. Ogata, Phys. Rev. Lett. 89, 116401 (2002).

10. T. Koretsune and M. Ogata, J. Phys. Soc. Jpn. 72, 2437 (2003).

11. Y. Matsumoto, D. Tsuji, S. Murakawa, H. Akisato, H. Kambara, and H. Fukuyama, J. Low. Temp. Phys. 138, 271 (2005).

12. D. Tsuji, Y. Matsumoto, S. Murakawa, H. Akisato, H. Kambara, and H. Fukuyama, J. Low. Temp. Phys. 138, 277 (2005).

13. D.N. Zubarev, Nonequilibrium Statistical Thermodynamics Consultant Bureau, New York (1974).

14. K. Ishida, M. Morishita, K. Yawata, and H. Fukuyama, Phys. Rev. Lett. 79, 3451 (1997). 